\title{
Dynamics of the Protrusion Domain of Herpes Simplex Virus Capsid from Time-Resolved Cryo-EM and Molecular Modelling.
}

\author{
J. Bernard Heymann*, Benes L. Trus*,** and Alasdair C. Steven* \\ * Laboratory of Structural Biology, NIAMS, NIH, Bethesda, MD 20892. \\ ** Imaging Science Laboratory, CIT, NIH, Bethesda, MD 20892.
}

The capsids of herpesviruses - a widely disseminated family of DNA animal viruses - are massive protein shells, $\sim 1250 \AA$ across and $\sim 192 \mathrm{MDa}$ in mass. Their surface shells are T=16 icosahedra with prominent cylindrical features, "protrusions", that extend $\sim 100 \AA$ radially outwards from the "floor" layer. Despite wide divergence in amino acid sequences and host range, herpesvirus capsids have a conserved architecture whose most variable feature is the morphology of their protrusions [1]. A crystal structure has been determined for the protrusion domain of herpes simplex virus type 1 (HSV1) [2]. The capsid is initially assembled as a precursor procapsid, which undergoes large structural changes during maturation [3]. In the procapsid, there is little contact between neighboring protrusion domains on a given hexon, whereas in the mature capsid they are closely associated in a 6-fold ring. The maturation pathway has been studied at a resolution of $\sim 20 \AA$ by time-lapse cryo-EM [3]. Here we attempt to combine cryo-EM data on the successive structural states with the high resolution structure of the protrusion domain to obtain a detailed account of its movements and interactions during maturation.

We docked the domain into the sequence of maps along the maturation pathway [3]. The general placement and orientations of the 16 non-symmetry-related VP5 domains per capsid are internally consistent for both the earliest procapsid and the fully mature capsid. The latter data are also consistent with an earlier analysis using a higher resolution reconstruction of the mature capsid [2]. In the procapsid, the protrusion domain fits snugly into the cryo-EM envelope indicating that its structure does not change significantly during maturation, i.e. it moves largely as a rigid body. Initially, adjacent protrusion domains on a given hexon make no contact with each other (Figs. 1, 2). As the capsid matures, they move progressively closer together. These motions involve varying magnitudes of rotation and relative translation, depending on the site on the capsid surface. For example, the penton-proximal hexon (P-hexon) starts out with a flattened asymmetric configuration of protrusion domains, which move towards a more compact, 6-fold symmetric, tightly associated ring (Fig. 2). The large movements undergone by the protrusion domains represent the amplification of shifts - rotations and translations - of the underlying floor domains to which the protrusion domains are attached. One consequence of protrusion remodelling is enhanced capsid stability. The interactions established between protrusion domains in hexons contribute to this stabilization. Nearest-neighbor contacts are not established to the same extent on pentons, rendering them the weakest points in the surface lattice and the most easily extracted [4].

1. B.L. Trus, et al., J. Virol. 75 (2001) 2879.

2. B.R. Bowman, et al., EMBO J. 22 (2003) 757.

3. J.B. Heymann, et al., Nat. Struct. Biol. 10 (2003) 334.

4. W.W. Newcomb, et al., J. Mol. Biol. 232 (1993) 499. 

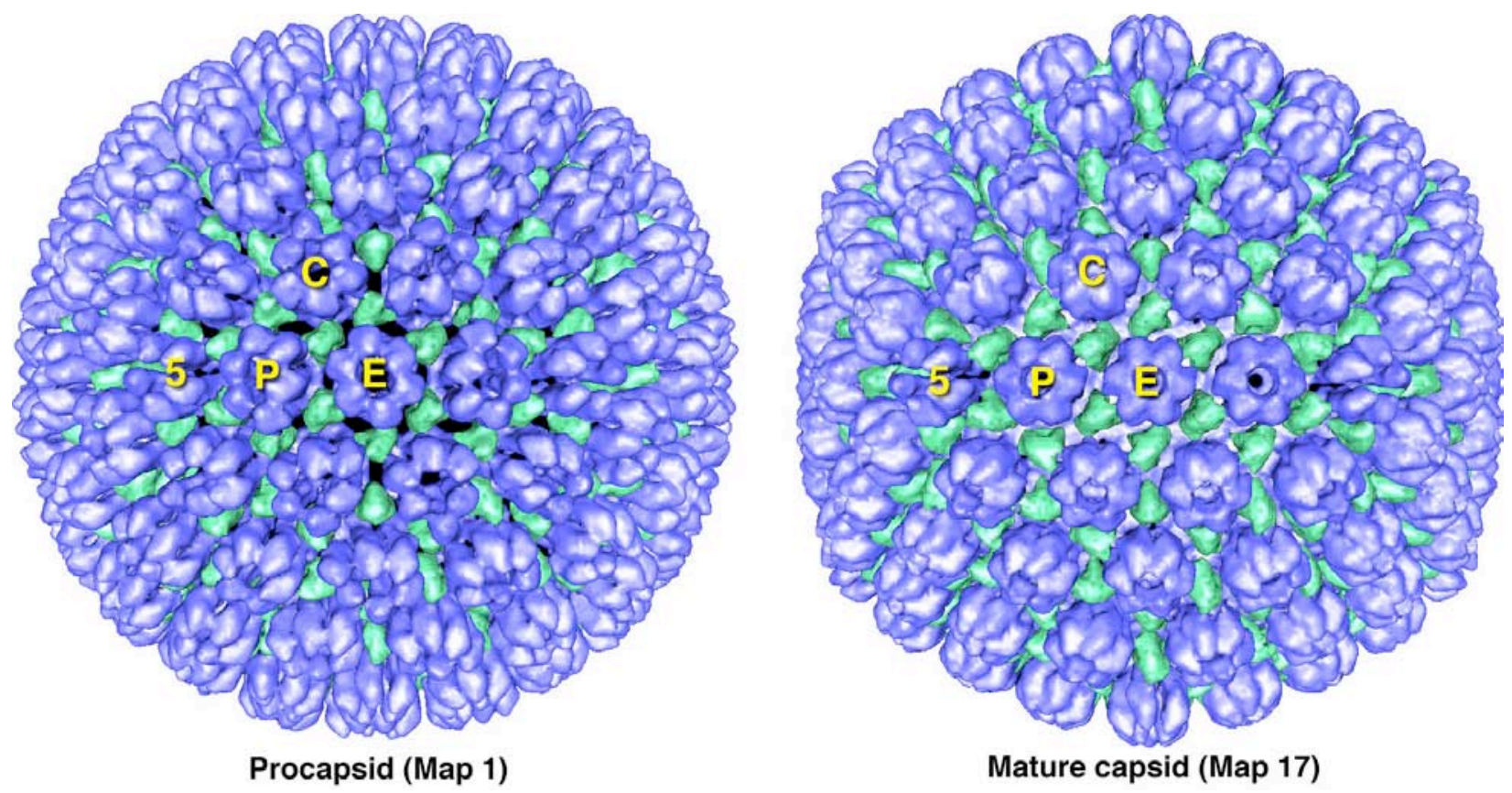

Figure 1: The procapsid and mature capsid maps of herpes simplex virus, showing the conversion from a spherical to a polyhedral particle, as well as the closing of the capsid shell floor (the black holes in the procapsid picture are replaced by the blue of the floor in the mature capsid). Blue: VP5; Green: triplexes (1 VP19c + 2 VP23 each); 5: penton; P: peripentonal hexon; E: edge hexon; C: central hexon. (Adapted from [3])
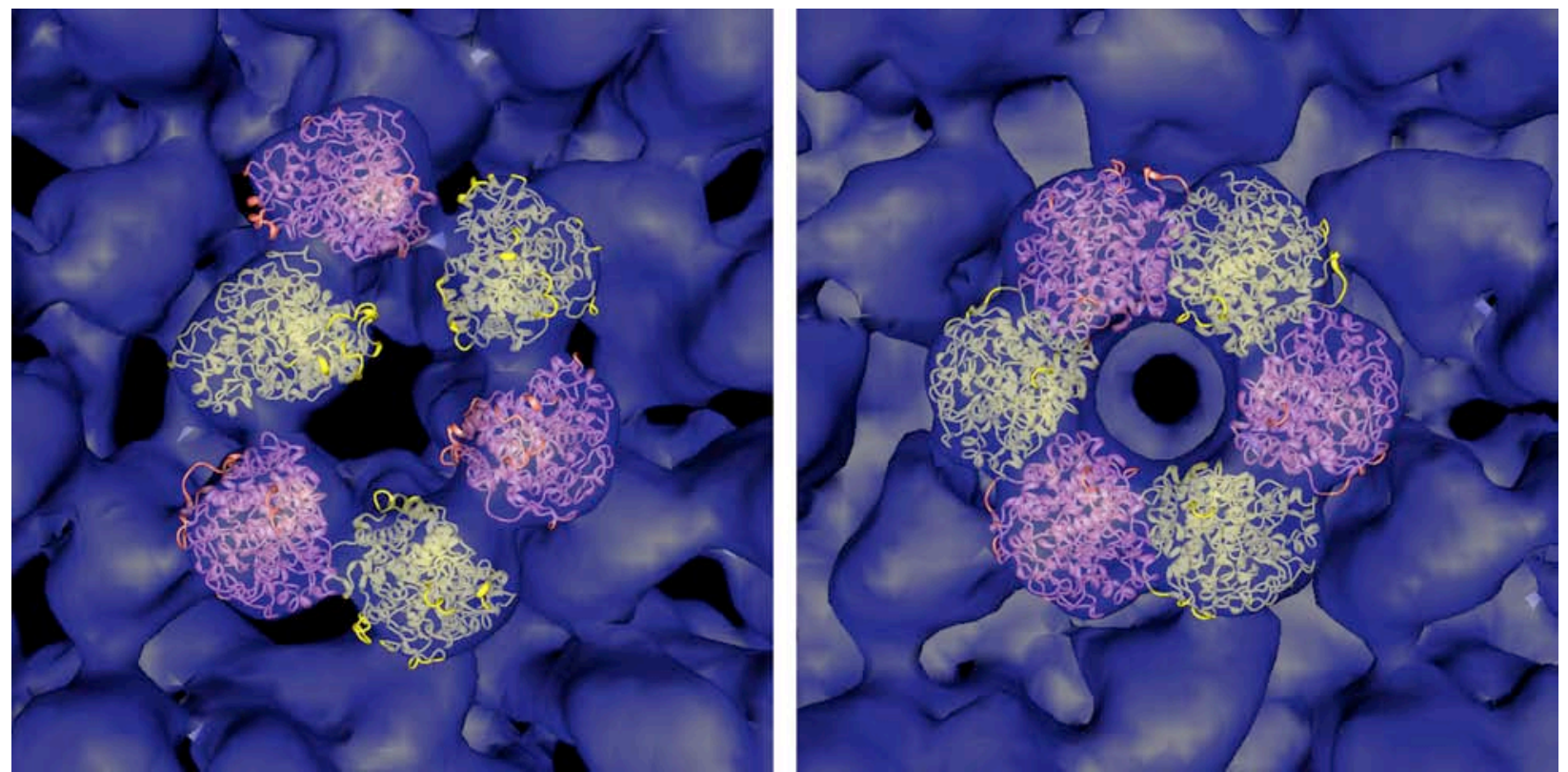

Figure 2: The protrusion domain atomic structure docked into the P-hexon subunits shows a clear separation in the procapsid between subunits, with significant translation and rotation during maturation to end in a condensed quasi-six-fold conformation in the mature P-hexon. 